This document is the preprint version of a published work that appeared in final form in Coll. Surf. $\mathrm{B}$, after peer review and technical editing by the publisher. To access the final edited and published work, see https://doi.org/10.1016/j.colsurfb.2018.04.044

\title{
Structural Organization of Lipid-Functionalized-Au Nanoparticles
}

\begin{abstract}
Alessandra Luchini ${ }^{\mathrm{a}}$, Gerardino D’Errico ${ }^{\mathrm{b}, \mathrm{c}}$, Serena Leone, ${ }^{\mathrm{b}}$ Zahra Vaezi ${ }^{\mathrm{d}}$, Annalisa Bortolotti $^{\mathrm{d}}$, Lorenzo Stella ${ }^{\mathrm{d}}$, Giuseppe Vitiello ${ }^{\mathrm{e}, \mathrm{c}, *}$ and Luigi Paduano ${ }^{\mathrm{b}, \mathrm{c}, *}$
\end{abstract}

${ }^{a}$ Niels Bohr Institute, University of Copenhagen, Universitet sparken 5, 2100, Copenhagen, Denmark

${ }^{\mathrm{b}}$ Department of Chemical Sciences, University of Naples “Federico II”, Complesso Universitario di Monte S. Angelo, via Cintia 4, 80126 Naples, Italy.

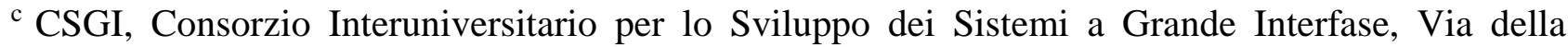
Lastruccia 3, 50019 Sesto Fiorentino (Florence), Italy.

${ }^{\mathrm{d}}$ Department of Chemical Sciences and Technologies, University of Roma Tor Vergata, Via della Ricerca Scientifica, 00133 Roma, Italy.

e Department of Chemical, Materials and Production Engineering, University of Naples “Federico II”, Piazzale Tecchio 80, 80125 Naples, Italy.

*Corresponding authors at: Department of Chemical, Materials and Production Engineering, University of Naples “Federico II”, Piazzale Tecchio 80, 80125 Naples, Italy and Department of Chemical Sciences, University of Naples Federico II, Complesso Universitario di Monte S. Angelo, via Cintia 4, 80126 Naples, Italy.

E-mail addresses: giuseppe.vitiello@unina.it (G. Vitiello), luigi.paduano@unina.it (L. Paduano).

\section{Statistical Summary}

Number of words: 4527

Number of figures/tables: 6 


\title{
Graphical Abstract
}

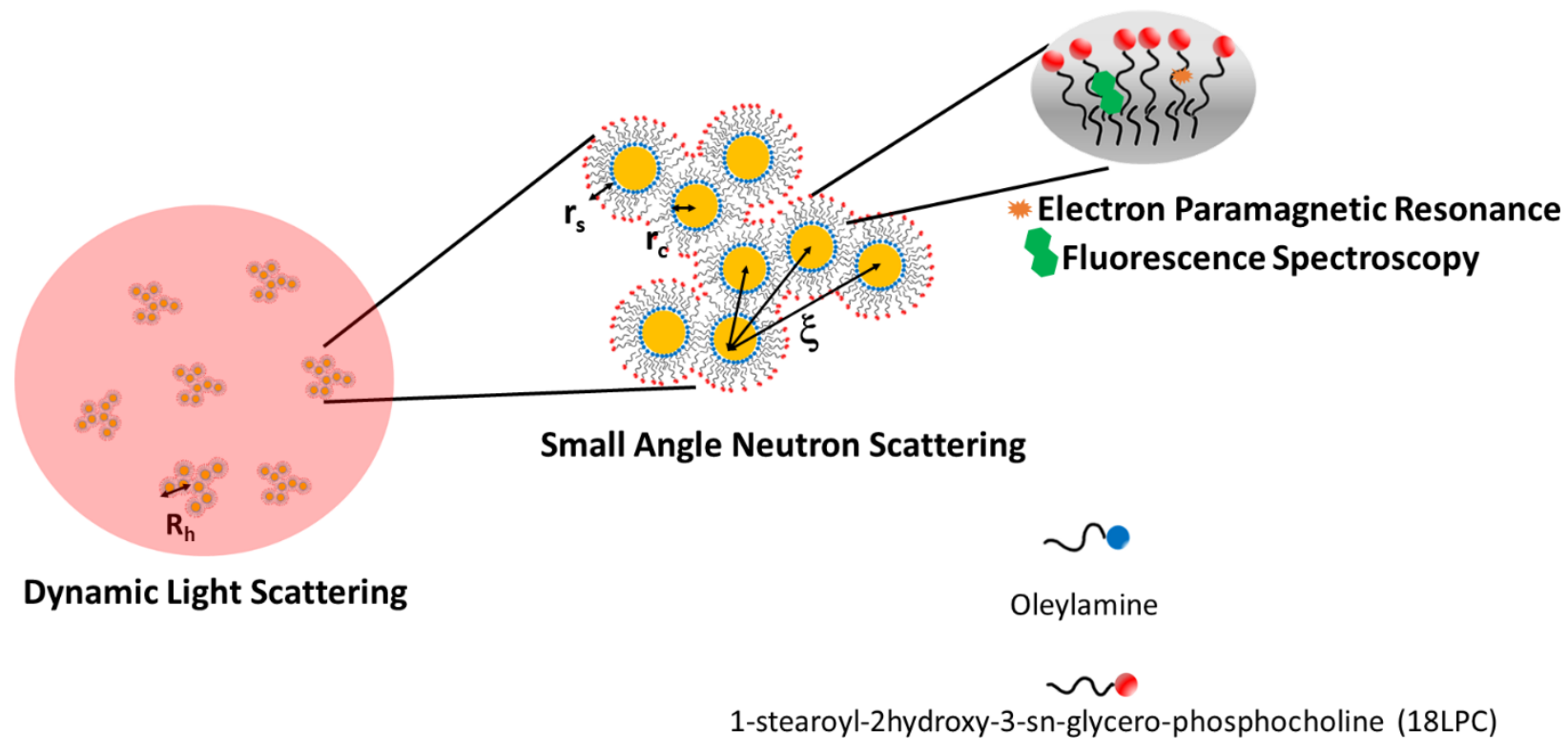

\begin{abstract}
Gold nanoparticles (AuNPs) are considered suitable systems for drug delivery and diagnostics with several applications in biomedicine. Size, shape and surface functionalization of these nanoparticles are important parameters influencing their behavior in a biological environment. This study describes the preparation and the characterization of lysophosphocholine coated AuNPs by means of Small Angle Neutron Scattering (SANS), Electron Paramagnetic Resonance (EPR) and Fluorescence Spectroscopy. In particular the structure of the functionalized AuNP suspension, as well as the physical properties, of the nanoparticle organic coating are discussed.

The experimental results indicated that functionalized lysophosphocholine-AuNPs form aggregates, which are composed by nanoparticles with core-shell structure. Nevertheless, the nanoparticle suspension resulted to be stable, without significant structural rearrangements even when the temperature was increased to $50{ }^{\circ} \mathrm{C}$. At the same time, experimental evidences also suggested that the 18LPC layer around AuNPs presented a reduced chain packing compared to pure 18LPC aggregates.
\end{abstract}

Keywords: gold nanoparticle, lipid, coating, amphiphiles. 


\section{Introduction}

Inorganic nanoparticles of different metals and metal oxides are used in nanomedicine, biotechnology, nanotechnology and electronics, catalysis and other industrial applications such as food packaging [3-5]. Several synthetic protocols were proposed to prepare inorganic nanoparticles (NPs) with suitable properties according to their final application [6-10]. However, there are still several concerns about the NP impact on the environment and on the human health. In the attempt to elucidate the actual toxicity of the NPs, many in vitro and in vivo studies were performed [11, 12]; as the main conclusion of these studies the physical properties of the NPs such as size, shape and surface functionalization appeared to be important parameters influencing their behavior in a biological environment $[13,14]$. In particular, the morphology and the composition of the shell coating the inorganic NP surface had a great impact on the interaction with biological systems, e.g. proteins and cell membranes [15-18].

Among the different kinds of nanoparticles, gold nanoparticles (AuNPs) were intensively studied [19, 20]. Historically, they were among the first types of NPs to be synthesized and nowadays several synthesis protocols are available in the literature to produce AuNPs with tunable size and shape [2123]. AuNPs are characterized by a poor stability in water suspension unless their surface is suitably coated. Different kinds of organic coatings were identified to preserve the colloidal stability of the nanoparticles and to improve their biocompatibility [29, 30]. Particularly interesting is the use of phospholipids to produce an amphiphilic coating on the NP surface [31, 32]. This type of functionalization was achieved by two different approaches: 1) the phospholipid molecules were introduced on the NP surface by hydrophobic interaction with a pre-formed coating layer of different composition, thus producing a hybrid bilayer; 2) the phospholipid molecules were used to coat bare NPs, which produced a symmetric bilayer on the NP surface [33]. Recently, approach 1) was implemented for successfully functionalizing NPs with lysophosphocholine (LPC) molecules [34]. LPC-functionalized NP suspensions are attractive systems according to the recent interest in the use of zwitterionic amphiphilic molecules to coat inorganic NPs for biomedical applications [35-37]. 
In this work, a physico-chemical approach based on the combination of scattering and spectroscopy techniques was used to characterize LPC-functionalized AuNPs. In particular, Small Angle Neutron Scattering (SANS) provided information on the overall structure of 18LPC/NPs in aqueous suspension. On the other hand, Electron Paramagnetic Resonance (EPR) and fluorescence spectroscopy provided complementary information on the morphology and of the 18LPC coating. 18LPC aggregates were used as a reference to compare the different self-assembly behavior of 18LPC molecules in water and in the functionalized AuNP suspension. 


\section{Materials and Methods}

\subsection{Materials}

Gold(III) chloride solution (99.99\% trace metals basis, $30 \mathrm{wt}$. \% in dilute $\mathrm{HCl}$ ), oleoylamine (OAM,C $\left.{ }_{9} \mathrm{H}_{18}=\mathrm{C}_{9} \mathrm{H}_{17} \mathrm{NH}_{2}, 70 \%\right)$, ethanol $(\geq 98 \%)$, hexane ( $\geq 98 \%$ ), spin-labelled $n$-doxyl-stearic acid ( $n$-DSA, $n=5$ and 16) with the nitroxide group at different positions, $n$, in the acyl chain, and 6Dodecanoyl- $N, N$-dimethyl-2-naphthylamine (LAURDAN, $\geq 97.0 \%$ ) were obtained from Sigma Aldrich. 1-stearoyl-2-hydroxy-3glicero-sn-phosphocholine (18LPC $\mathrm{C}_{26} \mathrm{H}_{54} \mathrm{NO}_{7} \mathrm{P}, \quad>99 \%$ ), was purchased from Avanti Polar Lipids (Birmingham, AL, USA) and stored at $-20{ }^{\circ} \mathrm{C}$.

\subsection{Synthesis and Functionalization of Au-NPs}

AuNPs were synthesized according to the method proposed by Choi et al [38]. In detail, $50 \mu \mathrm{L}$ of $\mathrm{HCl}$ solution of $\mathrm{HAuCl}_{4}(30 \% \mathrm{w} / \mathrm{w} \mathrm{HCl}$ and $17 \% \mathrm{w} / \mathrm{w} \mathrm{Au}(\mathrm{III}))$ and $1.5 \mathrm{~mL}$ of oleylamine were mixed together in a three-neck flask; in this protocol the oleylamine is used both as solvent and stabilizing agents for the nanoparticles. The solution was initially heated at $60{ }^{\circ} \mathrm{C}$ for 15 min under argon atmosphere and subsequently stirred at $120{ }^{\circ} \mathrm{C}$ for $30 \mathrm{~min}$ and then at $210{ }^{\circ} \mathrm{C}$ for $1 \mathrm{~h}$ (Supplementary Material, Figure S1). After $1.5 \mathrm{~h}$, the flask was removed from the heater and cooled to room temperature. The suspension was treated with $\sim 15 \mathrm{~mL}$ of ethanol and centrifuged at 5000 rpm for 10 min to remove the undesired components. Au-NPs coated with oleylamine were obtained as a solid precipitate and subsequently were dispersed in $5 \mathrm{~mL}$ of cyclohexane. AuNP suspension in cyclohexane was characterized by Dynamic Light Scattering (DLS). The results of this characterization are discussed in the Supplementary Material.

Stable aqueous suspensions of AuNPs were obtained upon functionalization with 18LPC molecules According to the recently proposed protocol [34, 40], $1 \mathrm{~mL}$ of AuNPs cyclohexane suspension was stratified over $1 \mathrm{~mL}$ of the 18LPC water solution $\left(4 \cdot 10^{-3} \mathrm{~m}\right.$ concentration). The resulting biphasic system was sonicated at $50{ }^{\circ} \mathrm{C}$ for about $2 \mathrm{~h}$ in order to promote cyclohexane evaporation and the 
transfer of the AuNPs in the aqueous phase, where they are stabilized by the 18LPC coating (named hereafter 18LPC/AuNPs). 18LPC molecules are hypothesized to orient themselves with the hydrophobic tail in the direction of the oleylamine layer, while the hydrophilic head is exposed toward the water. The adopted functionalization strategy does not require any purification step leading directly to the obtainment of the functionalized AuNPs.

\subsection{Small Angle Neutron Scattering (SANS)}

SANS measurements were performed on D22 at the Institut Laue Langevin (ILL), Grenoble (France). Neutrons with a wavelength spread $\Delta \lambda / \lambda \leq 0.2$ were used. A two-dimensional array detector at three different wavelength (W)/collimation (C)/sample-to-detector(D)/distance combinations ( $\mathrm{W}_{6 \AA} \mathrm{C}_{2.8 \mathrm{~m}} \mathrm{D}_{1.4 \mathrm{~m}}, \mathrm{~W}_{6 \AA} \mathrm{C}_{5.6 \mathrm{~m}} \mathrm{D}_{5.6 \mathrm{~m}}$ and $\mathrm{W}_{6} \mathrm{C}_{17.6 \mathrm{mD}} \mathrm{D}_{17.6 \mathrm{~m}}$ ) was used to measure neutrons scattered from the samples. These configurations allowed collecting data in a range of the scattering vector modulus $q=4 \pi / \lambda \sin (\theta / 2)$ between $0.002 \AA^{-1}$ and $0.59 \AA^{-1}$, with $\theta$ being the scattering angle. The investigated systems were contained in a closed quartz cell, in order to prevent the solvent evaporation. The raw data were then corrected for background and empty cell scattering.

The obtained absolute scattering cross sections $d \Sigma / d \Omega$ data were plotted as function of $q$. Generally, the dependence of $\mathrm{d} \Sigma / \mathrm{d} \Omega$ from the scattering vector can be summarized as in equation 1 .

$$
\frac{d \Sigma}{d \Omega}=\phi_{p} V_{p} P(q) S(q)+b k g
$$

where $\phi_{p}, V_{p}, P(q), S(q)$ represent the volume fraction of the particles, the particle volume, the form and the structure factor of the scattering particles respectively, while $b \mathrm{~kg}$ is the background, largely dependent on any hydrogen present. The form factor is responsible for the shape, size, size distribution of the scattering particles, while a contribution of the structure factor can be considered when an interparticle correlation exists. The structural information contained in both the form and the 
structure factor can be extrapolated by choosing an appropriate model to fit the obtained experimental data [41].

\subsection{Electron Paramagnetic Resonance (EPR)}

EPR spectra were recorded with a $9 \mathrm{GHz}$ Bruker Elexys E-500spectrometer (Bruker, Rheinstetten, Germany). The samples were transferred in capillaries and subsequently placed in a standard $4 \mathrm{~mm}$ quartz sample tube containing light silicone oil for thermal stability. All the measurements were performed at $25^{\circ} \mathrm{C}$. Spectra were recorded using the following instrumental settings: sweep width, 120 G; resolution, 1024 points; time constant, 20.48 ms; modulation frequency, $100 \mathrm{kHz}$; modulation amplitude, $1.0 \mathrm{G}$; incident power, $6.37 \mathrm{~mW}$. Several scans, typically 64, were accumulated to improve the signal-to-noise ratio. Samples for EPR measurements were prepared by adding $1 \% \mathrm{~mol} / \mathrm{mol}$ of $n$ DSA ( $n=5$ or 16 ), as spin-labels, to the 18LPC solution used for AuNPs functionalization. $n$-DSA are amphiphilic molecules, like to mono-chained lipids, bearing a radical nitroxide group close to the hydrophilic headgroup (5-DSA) or, alternatively, close to the terminus of the hydrophobic tail (16DSA). Whenever inserted in supramolecular aggregates formed by other amphiphiles, $n$-DSA monitors the structure and dynamics of the aggregate/aqueous medium interphase, also furnishing information about the microstructural organization of these amphiphiles. Particularly, 5-DSA shows an almost isotropic three-line spectrum when embedded in micelles [42, 43], while a well-resolved anisotropic line shape is observed for vesicles [43]. A quantitative analysis of $n$-DSA spectra for all lipid samples was realized determining the acyl chain order parameters relative to the bilayer normal, $S$, and the isotropic hyperfine coupling constants for the spin-labels in the membrane, $\boldsymbol{a}_{\mathrm{N}}^{\prime}$, through a home-made MATLAB-based software routine.

\subsection{Fluorescence Spectroscopy}


Fluorescence spectra were collected with a Fluoromax 4 (Horiba-JobinYvon, France) spectrofluorimeter. Temperature was controlled to $25{ }^{\circ} \mathrm{C}$ by a thermostat connected to the cuvette holder. Experiments were performed in 3x3 mm quartz cells, using the following conditions: excitation wavelength $365 \mathrm{~nm}$; emission wavelength 380-650 nm; data interval $1 \mathrm{~nm}$; bandwidth 2 $\mathrm{nm}$ in excitation and $3 \mathrm{~nm}$ in emission, integration time $1 \mathrm{~s}$.

Samples for fluorescence spectroscopy were prepared by adding the fluorophore to the 18LPC/AuNP suspension. In details, 18LPC/AuNPs suspension was diluted in water to a 18LPC concentration of $250 \mu \mathrm{M}$, and subsequently LAURDAN was added from a concentrated methanolic solution to a final concentration of $0.5 \mu \mathrm{M}$. The methanol concentration in the final sample was below $2 \%(\mathrm{v} / \mathrm{v})$, ensuring a negligible effect on the stability of the organic coating [44]. The concentration of the LAURDAN stock solution was determined by absorbance, using a molar extinction coefficient of $20000 \mathrm{M}^{-1} \mathrm{~cm}^{-1}$ at $364 \mathrm{~nm}[45]$.

\section{Results}

\subsection{SANS characterization}

SANS measurements were performed to investigate the structural organization of 18LPC/AuNPs at three different temperatures $\left(20^{\circ} \mathrm{C}, 37^{\circ} \mathrm{C}\right.$ and $50{ }^{\circ} \mathrm{C}$ ). Figure 1 (panel a) shows the collected experimental data together with the corresponding fitting curves. 


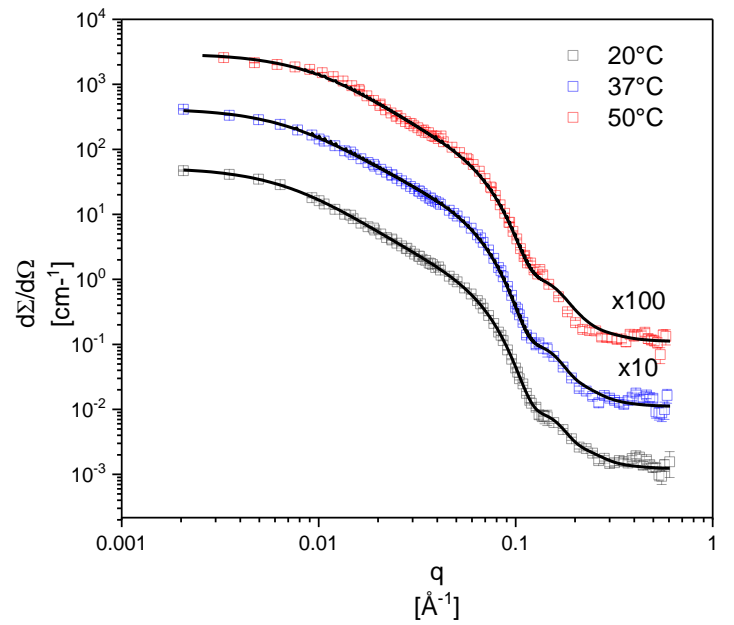

$\left[\AA^{-1}\right]$

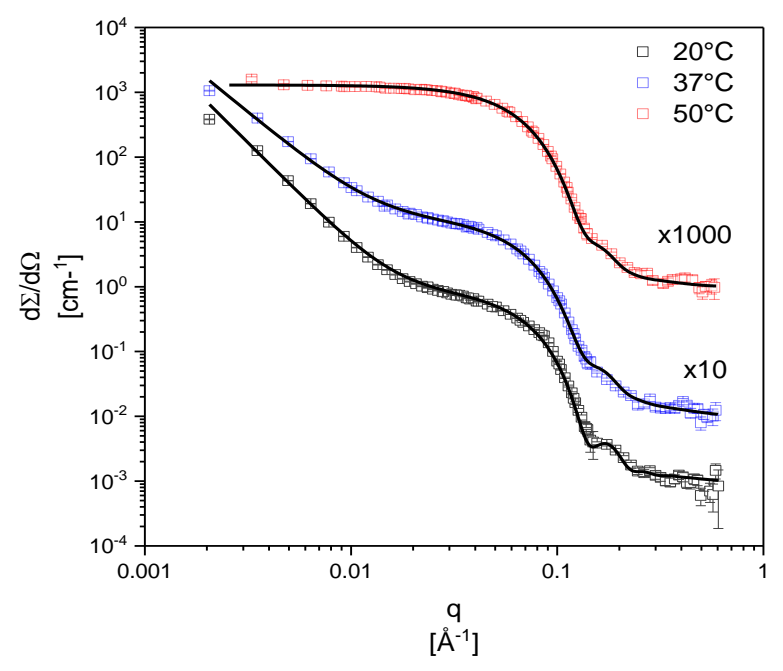

Figure 1 - SANS data collected for 18LPC/AuNPs in $\mathrm{D}_{2} \mathrm{O}$ (panel a) and 18LPC in $\mathrm{D}_{2} \mathrm{O}$ at $20^{\circ} \mathrm{C}, 37^{\circ} \mathrm{C}$ and $50{ }^{\circ} \mathrm{C}$ as reported in the legend.

Inspection of the curves in Figure 1 led to the observation that the data trend was overall very similar at the different temperatures, and consequently that 18LPC/AuNPs did not undergo any substantial structural change when the temperature was increased up to $50{ }^{\circ} \mathrm{C}$. This observation suggested relevant thermal stability of the system. The quantitative analysis of SANS data required the identification of a suitable model to describe the scattered intensity vs $q$ and to extract structural information on the sample. In this specific case, preliminary information available on the sample were used to identify different models, which were subsequently tested (see Supplementary Materials). Indeed, DLS measurements highlighted the presence of nanoparticle aggregates in solution with mean hydrodynamic radius $(700 \pm 80) \AA$. None of the explored models, which included a defined shape and total size of the nanoparticle aggregates could properly fit the experimental SANS data and be in agreement with the hydrodynamic radius obtained from DLS. This conclusion suggested that the aggregates were formed by randomly oriented nanoparticles. Nevertheless, information concerning the internal structure of the aggregate, i.e. isolated nanoparticle shape and size, were still contained in the collected data. 
NP aggregation is largely reported in the literature [34, 46, 47]; frequently the aggregates are characterized by a randomly oriented structure, which can be described mathematically as a fractal objects [48-50]. This description resulted to be the most appropriate to interpret the SANS data collected on 18LPC/AuNP suspension.

According to the model proposed by Teixeira [51], the intensity scattered by a fractal object can be described by the combination of a form and a structure factor, which respectively describes the shape of the repetitive unit and the interparticle interaction in the fractal. Specifically, SANS data were analysed according to equation 1 with the form factor $(P(q))$ and the structure factor $(S(q))$ reported in equation 2 and 3 respectively.[52]

$$
\begin{gathered}
P_{\text {core-shell }}(q)=\frac{1}{V^{2}}\left[\begin{array}{c}
3 V_{\text {core }}\left(\rho_{\text {core }}-\rho_{\text {shell }}\right) \frac{\left[\sin \left(q r_{\text {core }}\right)-q r_{\text {core }} \cos \left(q r_{\text {core }}\right)\right]}{\left(q r_{\text {core }}\right)^{3}}+ \\
+3 V_{\text {shell }}\left(\rho_{\text {shell }}-\rho_{D_{2} O}\right) \frac{\left[\sin \left(q r_{\text {shell }}\right)-q r_{\text {shell }} \cos \left(q r_{\text {shell }}\right)\right]}{\left(q r_{\text {shell }}\right)^{3}}
\end{array}\right] \\
S(q)=1+\frac{D \Gamma(D-1)}{\left[1+\frac{1}{(q \xi)^{2}}\right]^{\frac{D-1}{2}} \frac{\sin \left[(D-1) \tan ^{-1}(q \xi)\right]}{\left(q r_{N P}\right)^{D}}}
\end{gathered}
$$

In this description the fractal structure was composed by aggregated 18LPC/AuNPs, which were represented as spheres with core-shell structure. $V_{\text {core }}$ and $V_{\text {shell }}$ were respectively the volumes of the gold core and organic shell of the nanoparticles with $r_{\text {core }}$ being the core radius and $r_{\text {shell }}$ being organic coating thickness. $\rho_{\text {core, }} \rho_{\text {shell }}$ and $\rho_{\text {solv }}$ were respectively the scattering length density of the nanoparticle core and shell and the solvent. This latter was fixed to $6.34 \cdot 10^{-6} \AA^{-2}$, which corresponded to the scattering length density of $\mathrm{D}_{2} \mathrm{O}$. On the other hand, the scattering length density of 18LPC/AuNPs was initially calculated on the grounds of the scattering length density of each

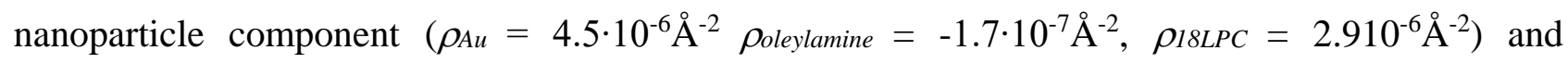
subsequently optimized during data fitting. 
While $P(q)$ takes into account the shape of 18LPC/AuNPs within the aggregates, $S(q)$ describes how they were arranged in space with respect to each other. In equation 3, $D$ is the Hausdorff dimension or the fractal dimension, and $\xi$ is the correlation length. This latter represents a characteristic length in the fractal structure, which corresponded as the interparticle distance within the aggregate.

The above described model resulted to efficiently describe the collected SANS curves and the structural parameters obtained from data analysis are summarized in Table 1.

As for the 18LPC/AuNP suspension, data were collected at the three temperatures $20^{\circ} \mathrm{C}, 37^{\circ} \mathrm{C}$ and $50{ }^{\circ} \mathrm{C}$ also in the case of 18LPC suspension. These experiments were performed to compare the behavior of the two systems as function of the temperature.

As reported in Figure1, panel b, 18LPC aggregates exhibited evident structural changes at temperatures higher than $20^{\circ} \mathrm{C}$. Indeed, in the high q-range $\left(0.04-0.60 \AA^{-1}\right)$ the three curves showed similar trend, which was indicative of the presence of small aggregates. On the other hand, in the low $q$ range $\left(0.015-0.002 \AA^{-1}\right)$, the curves referring to $20^{\circ} \mathrm{C}$ and $37^{\circ} \mathrm{C}$ clearly exhibited a large increase of the scattered intensity, while at $50{ }^{\circ} \mathrm{C}$ a constant scattered intensity value was measured. These observations suggested that large aggregates are present only at $20^{\circ} \mathrm{C}$ and $37^{\circ} \mathrm{C}$.

At room temperature, 18LPC molecules are known to form in aqueous medium both micelles and large lamellar structures [53-55]. By increasing the temperature, the exclusive formation of micelles in solution is promoted $[53,56]$. Hence, the experimental data at $20^{\circ} \mathrm{C}$ and $37^{\circ} \mathrm{C}$ were analyzed by summing two form factors accounting for the 18LPC micelles and the larger lamellar structures, while a single form factor was used to analyze the data at $50{ }^{\circ} \mathrm{C}$. The scattered intensity raised by the $18 \mathrm{LPC}$ micelles was treated according to equation 1 with the form factor reported in equation 2 and structure factor corresponding to 1 . In the case of 18LPC micelle, the subscripts "core" and "shell” in equation 2 referred respectively to the hydrophobic core composed by the 18LPC acyl chains and the hydrophilic outer shell composed of 18LPC polar headgroups.

The intensity scattered by the large lamellar structure was modelled as a power law, equation 4 .

$$
I(q) \approx q^{-m}+b k g
$$


A suitable model to analyse scattering data from multilamellar structure is the lamellar paracrystal model proposed by Bergstrom and co-workers [57]. In the present case a simpler model as the sum of equation 2 and 4 was adopted to avoid overloading of fitting parameters in the model. Nevertheless, by comparing the slope of the scattered intensity in the low-q region ( $m$, power law exponent) for the data collected at $20^{\circ} \mathrm{C}$ and $37^{\circ} \mathrm{C}$ (Table 1), the smaller value of $m$ at $37^{\circ} \mathrm{C}$ might imply a reduced number of lamellae in the 18LPC large aggregates.

Table 1 - Structural parameters optimized from SANS data fitting for 18LPC and 18LPC/AuNP suspensions at $20^{\circ} \mathrm{C}, 37^{\circ} \mathrm{C}$ and $50{ }^{\circ} \mathrm{C}$. The volume fraction of the NPs resulted to be $0.30 \pm 0.05$ at all the explored temperatures.

\begin{tabular}{|c|c|c|c|}
\hline $\mathbf{1 8 L P C}$ & $\mathbf{T}=\mathbf{2 0}{ }^{\circ} \mathbf{C}$ & $\mathbf{T}=\mathbf{3 7}{ }^{\circ} \mathbf{C}$ & $\mathbf{T}=\mathbf{5 0}{ }^{\circ} \mathbf{C}$ \\
\hline Micellar-core radius $(\AA)$ & $22 \pm 3$ & $23 \pm 1$ & $22 \pm 2$ \\
\hline Micellar-shell thickness $(\AA)$ & $9 \pm 2$ & $9 \pm 4$ & $10 \pm 3$ \\
\hline micelle polydispersity & $0.15 \pm 0.01$ & $0.2 \pm 0.1$ & $0.2 \pm 0.1$ \\
\hline Power law slope & $3.0 \pm 0.2$ & $2.6 \pm 0.1$ & --- \\
\hline $\mathbf{1 8 L P C / A u N P s}$ & $\mathbf{T}=\mathbf{2 0}{ }^{\circ} \mathbf{C}$ & $\mathbf{T}=\mathbf{3 7}{ }^{\circ} \mathbf{C}$ & $\mathbf{T}=\mathbf{5 0}{ }^{\circ} \mathbf{C}$ \\
\hline NP core radius $(\AA)$ & $8 \pm 3$ & $9 \pm 2$ & $8 \pm 3$ \\
\hline NP shell thickness $(\AA)$ & $24 \pm 2$ & $27 \pm 3$ & $24 \pm 2$ \\
\hline NP polydispersity & $0.23 \pm 0.05$ & $0.18 \pm 0.03$ & $0.28 \pm 0.05$ \\
\hline Correletion length $(\AA)$ & $133 \pm 5$ & $137 \pm 7$ & $94 \pm 5$ \\
\hline Fractal dimension & $2.2 \pm 0.2$ & $2.0 \pm 0.1$ & $2.3 \pm 0.3$ \\
\hline
\end{tabular}

\subsection{EPR analysis}

Changes in lipid supramolecular ordering and dynamics in 18LPC/AuNPs were investigated by analyzing the EPR spectra of DSA molecules spin-labelled on the $n$ C-atom of the acyl chain ( $n$-DSA, $n=5$ and 16) which were incorporated in the 18LPC coating (1\% by weight on total lipids). EPR spectra of 5-DSA and 16-DSA in the lipid coating of AuNPs are shown in Figure 2. As reference, the EPR spectra of 5-DSA and 16-DSA in pure 18LPC lipid aggregates are also reported. Particularly, 5DSA spectra present a clearly defined axially anisotropic lineshape, as generally observed for vesicles [43]. On the other hand, the higher isotropy of the 16-DSA spectra with respect to those obtained for 
5-DSA indicates an increased flexibility in the segmental chain mobility, going from the polar headgroups to the inner hydrophobic core.
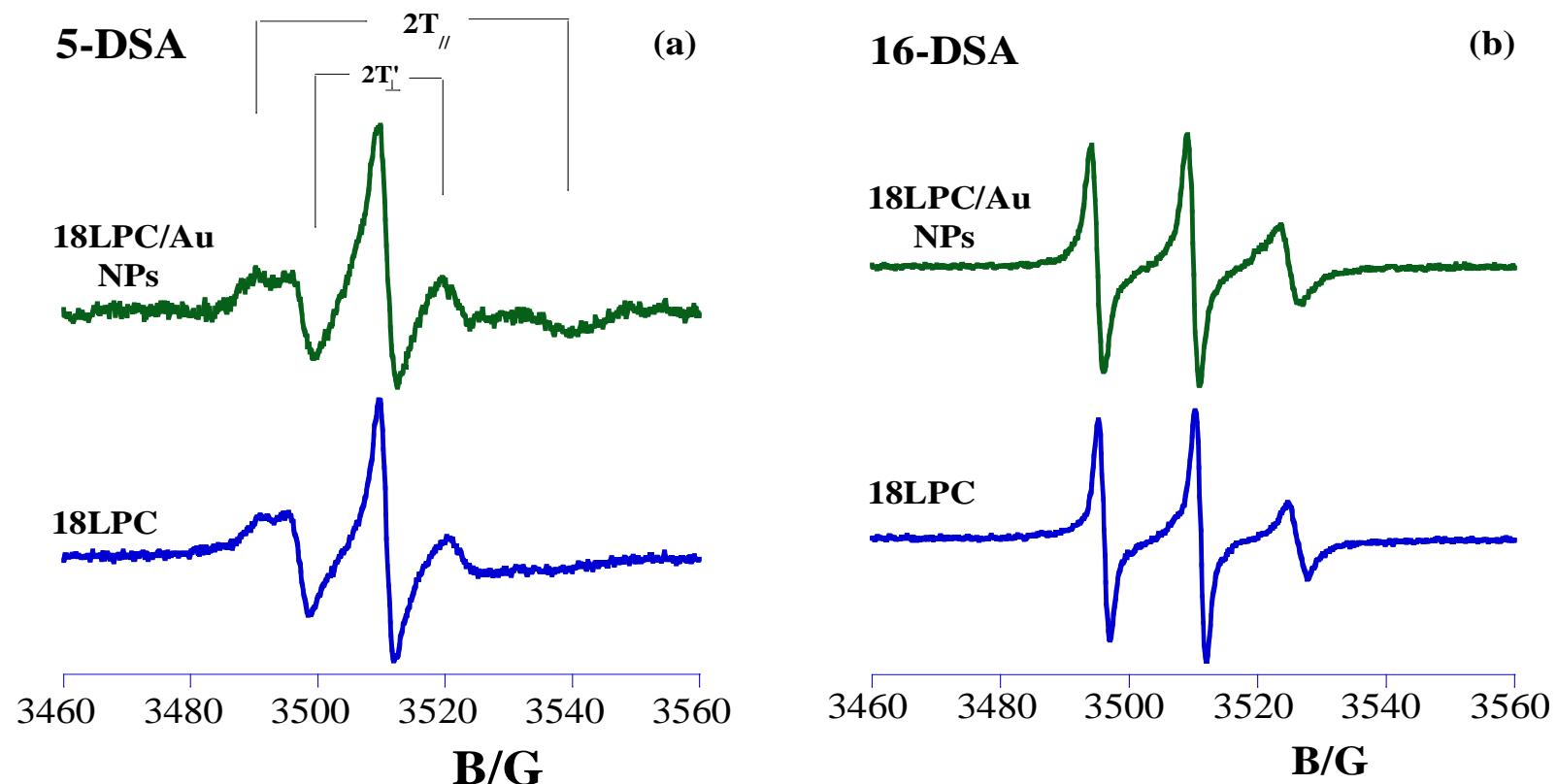

Figure 2 - EPR spectra of 5-DSA (panel a) and 16-DSA (panel b) inserted into the 18LPC external layer of the coating on the AuNPs surface.

The EPR spectra lineshape is also influenced by both the local polarity experienced by the label and its rotational mobility, as determined by the microenvironment viscosity and the structural order. In an attempt to quantitatively analyze the spectra, the order parameter, $S$, and the isotropic hyperfine coupling constant, $a_{N}^{\prime}$ were determined. $S$ is related to the angular amplitudes of the label motion, which in turn reflects the motion of the acyl chain segment to which the label is bound. On the other hand, the isotropic hyperfine coupling constant is an index of the micropolarity experienced by the nitroxide; in particular, it increases with the environmental polarity. $S$ and $a_{N}^{\prime}$ were calculated according to the relations:

$$
\begin{aligned}
& a_{N}^{\prime}=\frac{1}{3}\left(T_{z z}+2 T_{x x}\right) \\
& S=\frac{\left(T_{/ /}+T_{\perp}\right)}{\left(T_{\mathrm{zz}}+T_{\mathrm{xx}}\right)} \frac{a_{N}}{a_{N}^{\prime}}
\end{aligned}
$$


where $\mathrm{T}_{\|}$and $\mathrm{T}_{\perp}$ are two phenomenological hyperfine splitting parameters, which can be determined from the spectrum as shown in Figure 2, panel a(note that $\left.2 \mathrm{~T}_{\perp}^{\prime}=2 \mathrm{~T}_{\perp}-1.6\right) \cdot \mathrm{T}_{\mathrm{xx}}$ and $\mathrm{T}_{\mathrm{zz}}$ are the principal elements of the real hyperfine splitting tensor in the spin Hamiltonian of the spin-label, which can be measured from the corresponding single-crystal EPR spectrum and are reported in the literature $\left(\mathrm{T}_{\mathrm{xx}}\right.$ $=6.1 \mathrm{G}$ and $\left.\mathrm{T}_{\mathrm{zz}}=32.4 \mathrm{G}\right) . a_{N}$ is the isotropic hyperfine coupling constant for the spin-label in crystal state, given by:

$$
a_{N}=\frac{1}{3}\left(T_{z z}+2 T_{x x}\right)
$$

The $a_{N} / a_{N}^{\prime}$ ratio in Eq. (7) corrects the order parameter for polarity differences between the crystal state and the membrane. To obtain reliable values of the $T_{\|}$and $T_{\perp}$ splittings, we adopted a homemade, MATLAB-based software routine.

By observing Figure 2, it can be noted that 5-DSA spectrum of 18LPC/AuNP system presents a higher grade of anisotropy than 18LPC, which tends to form both micelles and lamellar phases at room temperature. This comparison suggests that the lipid coating in 18LPC/AuNPs is characterized by a more compact microstructure $[58,59]$ with respect to 18LPC aggregates. A similar behavior is also observed for 16-DSA spectra shown in Figure 2 panel b, confirming a different organization of lipids in 18LPC/AuNPs with respect to pure 18LPC aggregates. This evidence is confirmed by $S$ and $a_{N}^{\prime}$ values reported in Table 2 .

Table $2-S$ and $a_{N}^{\prime}$ values obtained from EPR spectra of 5-DSA and 16-DSA inserted into 18LPC aggregates and 18LPC lipid coating on the Au-NPs surface.

\begin{tabular}{|l|c|c|c|c|}
\hline \multirow{2}{*}{} & \multicolumn{2}{|c|}{ 5-DSA } & \multicolumn{2}{c|}{ 16-DSA } \\
\cline { 2 - 5 } & $\boldsymbol{S}$ & $\boldsymbol{a}_{\boldsymbol{N}}^{\prime}$ & $\boldsymbol{S}$ & $\boldsymbol{a}_{\boldsymbol{N}}^{\prime}$ \\
\hline 18LPC/AuNPs & $0.51 \pm 0.02$ & $15.6 \pm 0.1$ & $0.10 \pm 0.02$ & $15.1 \pm 0.1$ \\
\hline 18LPC & $0.42 \pm 0.02$ & $15.7 \pm 0.1$ & $0.07 \pm 0.02$ & $15.2 \pm 0.1$ \\
\hline
\end{tabular}




\subsection{Fluorescence analysis}

The organic coating of AuNPs was also investigated by fluorescence spectroscopy, using 6-lauroyl2-(dimethylamino)-naphtalene (LAURDAN). This fluorophore inserts spontaneously in lipidic phases and senses the level of hydration and water dynamics[60]. In phospholipid bilayers, its sensitivity to solvation causes a red shift of approximately $50 \mathrm{~nm}$ in the emission spectrum when membranes show a phase transition from a gel to a liquid state[61]. The shape of the LAURDAN emission spectrum is often summarized by a simple parameter termed general polarization [61](GP) (by formal analogy to fluorescence polarization) and defined as

$$
G P=\frac{I_{B}-I_{R}}{I_{B}+I_{R}}
$$

where $\mathrm{B}$ and $\mathrm{R}$ correspond to the intensities measured at wavelengths corresponding to the blue and red part of the emission spectrum, i.e. $435 \mathrm{~nm}$ and $485 \mathrm{~nm}$, respectively. GP can be considered as a measure of the hydration and the dynamics of the lipid milieu; in phospholipid bilayers, positive values correspond to membranes in the gel phase, while negative values are observed for fluid bilayers.

In the fluorescence experiments, LAURDAN was added to the 18LPC/AuNP suspension and the spectrum was followed over time (Figure 3). The spectra were observed to blue-shift with a relatively slow kinetics, in the time-frame of about one hour. This observation clearly indicated a slow probe insertion in the organic layers covering the nanoparticles. A similar trend was observed also for 18LPC aggregates, but in that case the kinetics of insertion was significantly faster[61] .

Both the spectral shape and the slow insertion kinetics probably due to the NP organic coating being less accessible as the nanoparticles are forming aggregates. This conclusion was further confirmed by fluorescence quenching experiments, where the effect on LAURDAN emission of a water-soluble quencher (KI) was measured to characterize the exposure of the probe to the aqueous phase. Figure 4 reports a Stern-Volmer plot, i.e. the ratio of $F_{0}$ (the fluorescence intensity in the absence of the quencher) and $\mathrm{F}$ (the fluorescence intensity measured at a given quencher concentration) as a function 
of KI concentration, showing that LAURDAN was essentially unaffected by the addition of KI, further supporting a deep insertion of the probe in a well packed environment.
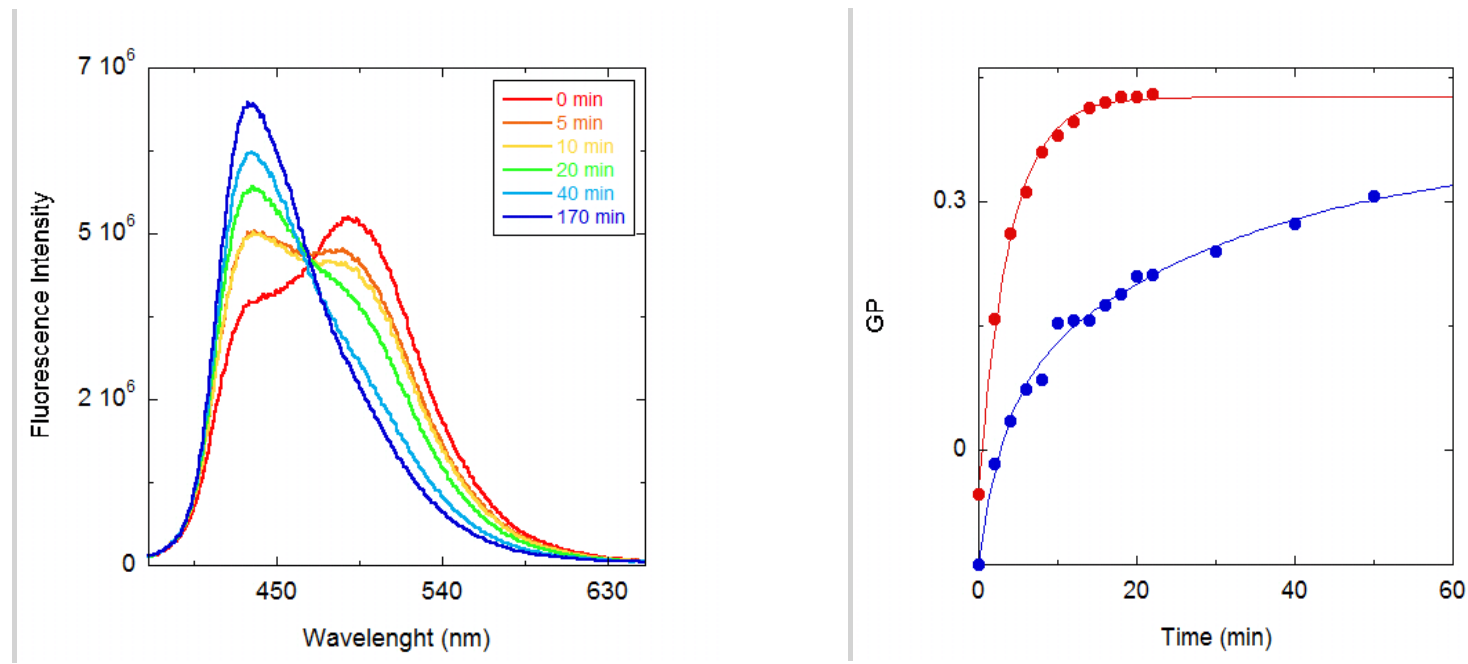

Figure 3 - Emission spectra of LAURDAN in 18LPC/AuNPs (panel a) and LAURDAN GP values measured in 18LPC (red line) and in 18LPC/AuNPs (blue line) as a function of time (b). The spectra were acquired under the following conditions: excitation $365 \mathrm{~nm}$; emission 380-650 nm; data interval $1 \mathrm{~nm}$; bandwidth $2 \mathrm{~nm}$ in excitation and 3nm in emission. [18LPC $]_{\mathrm{Au}-\mathrm{NPs}}=250 \mu \mathrm{M},[18 \mathrm{LPC}]=250 \mu \mathrm{M}$, [Laurdan $]=0.5 \mu \mathrm{M}$.

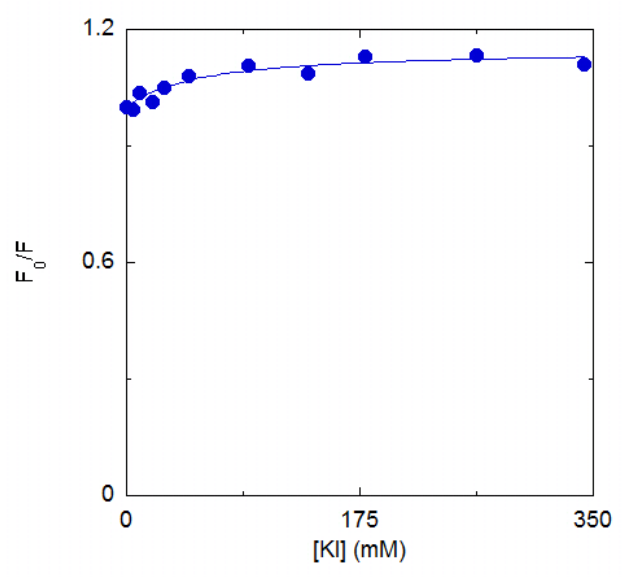

Figure 4 - Stern-Volmer plot of the quenching of Laurdan in 18LPC/AuNPs by potassium iodide[18LPC $]_{\mathrm{Au}-}$ $\mathrm{NPs}=250 \mu \mathrm{M}$, [Laurdan $]=0.5 \mu \mathrm{M},[\mathrm{KI}]$ from 0 to $350 \mathrm{mM}$. 


\section{Discussion}

The organic coating of functionalized nanoparticles represents the most external nanoparticle interface and thus its physical properties play a fundamental role in the interaction between the NPs and the surrounding environment $[17,62,63]$. Here, the characterization the 18LPC/AuNP structure and of the main features of their organic coating was described.

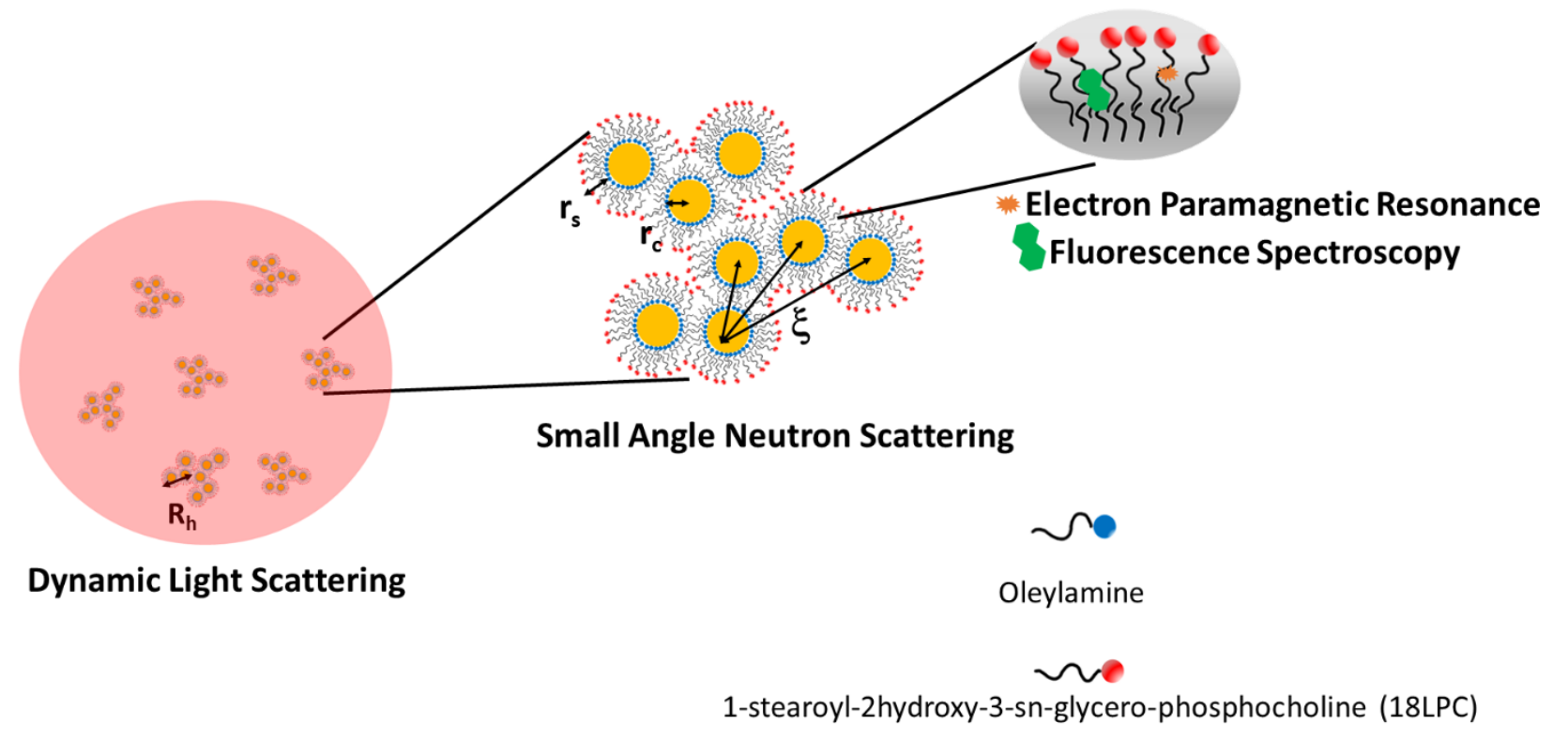

Figure 5 - Schematic representation the 18LPC/AuNP suspension characterization by different scattering and spectroscopy techniques. Dynamic Light Scattering (DLS) was used to estimate the total aggregate size $\left(\mathrm{R}_{\mathrm{h}}\right)$, Small Angle Neutron Scattering (SANS) was used to characterize nanoparticle gold radius $\left(r_{c}\right)$ and organic shell thickness $\left(\mathrm{r}_{\mathrm{s}}\right.$ ) as well as the mean interparticle distance $\xi$. Electron Paramagnetic Resonance (EPR) and Fluorescence Spectroscopy were used to characterize the dynamics of the nanoparticle organic coating. The relevant structural quantities obtained by DLS and SANS are reported in the schematic pictures.

In particular, functionalized AuNPs were prepared by first synthesizing AuNPs coated by oleylamine molecules, which are used as stabilizing agents during nanoparticle synthesis, and subsequently producing a second layer of 18LPC molecules around the gold core as detailed in the Materials and Method section. In order to define the structure of the synthesized nanoparticles, different scattering techniques were combined. Dynamic Light Scattering (DLS) was initially used to probe the overall size of both the synthesis and functionalization products (respectively AuNPs coated with oleylamine and 18LPC/AuNPs, i.e. AuNPs coated with both oleylamine and 18LPC molecules). As also 
confirmed by the SANS characterization, while the synthesis product was composed by small nanoparticle aggregates, $60 \pm 10 \AA ̊$ hydrodynamic radius, upon functionalization with 18LPC larger aggregates with (700 \pm 80) Å hydrodynamic radius were formed in solution.

NP aggregation is typically promoted by the nanoparticles reaching a lower surface energy within the aggregate compared to the single NPs in suspension. In the present case, it is reasonable to hypothesize the aggregate formation mainly occurred during AuNPs functionalization; the positive hydrophobic interaction between oleylamine chains might promote nanoparticle aggregation during 18LPC coating formation.

Even if the nanoparticle suspension was characterized by the presence of aggregates, detailed information on the single nanoparticle structure were still accessible. Indeed, SANS data were successfully analyzed by describing the 18LPC/AuNPs within the aggregates as core-shell spheres with (10 \pm 5) $\AA$ and (26 \pm 5$) \AA$ for the gold core radius and organic shell thickness respectively (Figure 5). 18LPC/AuNPs are the building block units of the aggregates, which were characterized by a nanoparticle volume fraction of $0.30 \pm 0.05$. The presence of an organic shell of $\sim 26 \AA$ around the gold core suggest that most of the nanoparticle surface is still coated by 18LPC within the aggregates.

On the ground of the available structural information, two different structures can be hypothesized for the NPs within the aggregate: 1) AuNPs with the surface fully coated by oleylamine molecules in the inner layer and 18LPC molecules in the outer layer are the building blocks of the aggregates; 2) AuNPs with the outer layer partially occupied by 18LPC molecules are the building blocks of the aggregate. Due to the potential repulsive interaction between AuNPs with the surface fully occupied by 18LPC molecules, the second structure might be the most relevant. According to this latter, the areas of the AuNP surface, which were not efficiently protected from the aqueous solvent by the additional 18LPC layer, would prevent the exposure of the oleylamine chains by aggregating with other nanoparticles. Hence, the AuNP surface area not covered by 18LPC molecules would represent the junctions within the aggregates where the oleylamine chains on different nanoparticles can be 
stabilized by positive interaction with each other. This interpretation of the 18LPC/AuNP aggregate structure is schematically reported in Figure5.

The model used to analyse the SANS data provided as well information about the inter-nanoparticle distance $(\xi)$ within the aggregates. Indeed, while the structure of the single nanoparticles composing the aggregate was unaffected by the temperature raise, at $20^{\circ} \mathrm{C}$ and $37^{\circ} \mathrm{C}$ the estimated mean distance between the nanoparticles within the aggregates was $(130 \pm 10) \AA$; at $50^{\circ} \mathrm{C}$, this distance resulted to be reduced to $(94 \pm 5) \AA$. Hence, increasing the temperature above $37^{\circ} \mathrm{C}$ can reduce the mean internanoparticle distance, but those not affect the structural parameters of the nanoparticles.

On the other hand, SANS data collected on the pure 18LPC suspension confirmed the temperatureinduced rearrangement of 18LPC aggregates as already reported in the literature [53]. 18LPC forms both micelles and larger aggregates with lamellar structures both at $20^{\circ} \mathrm{C}$ and $37^{\circ} \mathrm{C}$, while at $50{ }^{\circ} \mathrm{C}$ only micelles are detected in the sample.

More detailed information on the structure and dynamics of the 18LPC coating were collected by means of EPR and fluorescence spectroscopy measurement on 18LPC/AuNP suspension. Both the techniques required the use of a suitable probe, radical-labelled lipids for EPR and LAURDAN for fluorescence spectroscopy, to investigate the coating layer (Figure 5). Also in this case, 18LPC aggregates were chosen as reference. EPR and fluorescence spectroscopy consistently highlighted that the external 18LPC coating has higher packing degree, and hence a reduced fluidity with respect to the 18LPC aggregates. This evidence was confirmed by both the higher order parameter calculated from the EPR spectra, and was particularly evident in the case of the 5DSA probe, and in the slower insertion of the LAURDAN 18LPC/AuNPs coating with respect to 18LPC aggregates.

\section{Conclusions}

In the view of biomedical application of suitably functionalized inorganic nanoparticles, detailed information on their structure are required to fully understand their interaction with biological system and avoid undesired cytotoxic effects. AuNPs can represent suitable candidates for both drug delivery and diagnostics, if a suitable coating is provided on their surface. 
In the presented work, the preparation of LPC-coated AuNPs, which could represent promising candidates for biomedical application, was described. More specifically, the collected SANS data showed that 18LPC/AuNPs formed aggregates in water suspension. Nevertheless, the nanoparticles within the aggregates were still characterized by a core-shell structure, which confirms the coating of the AuNPs by the amphiphilic molecules. Furthermore, the system is characterized by good stability even at high temperature, i.e. $50{ }^{\circ} \mathrm{C}$. The information about the nanoparticle structure obtained by SANS were complemented by EPR and fluorescence spectroscopy experiments. Both the techniques highlighted that the organization of 18LPC molecules around AuNPs leads to an external coating with an higher packing degree and reduced fluidity with respect to pure phosphocholine aggregates. The results here discussed represent a useful reference in the field of phospholipid-functionalized inorganic nanoparticles and provide a relevant example of how the structure of complex system, such as functionalized nanoparticle suspension, can be determined on different levels by combining scattering and spectroscopy techniques.

\section{Acknowledgements}

The authors thank the Institut Laue-Langevin for award of beamtime and use of the support facilities in the Partnership for Soft Condensed Matter. The authors thank Dr. Lionel Porcar for help during the SANS experiment. This work benefited from the use of the SasView application, originally developed under NSF award DMR-0520547. SasView contains code developed with funding from the European Union's Horizon 2020 research and innovation programme under the SINE2020 project, grant agreement No 654000. 


\section{References}

[1] R.J. Hamers, Nanomaterials and Global Sustainability, Acc Chem Res, 50 (2017) 633-637.

[2] H. Huang, J.F. Lovell, Advanced Functional Nanomaterials for Theranostics, Adv Funct Mater, 27 (2017).

[3] V.M. Rotello, Interfacing Inorganic Nanoparticles with Biology, Bioconjug Chem, 28 (2017) 12.

[4] S. Fogli, C. Montis, S. Paccosi, A. Silvano, E. Michelucci, D. Berti, A. Bosi, A. Parenti, P. Romagnoli, Inorganic nanoparticles as potential regulators of immune response in dendritic cells, Nanomedicine (Lond), 12 (2017) 1647-1660.

[5] J.A. Darr, J. Zhang, N.M. Makwana, X. Weng, Continuous Hydrothermal Synthesis of Inorganic Nanoparticles: Applications and Future Directions, Chem Rev, 117 (2017) 11125-11238.

[6] S. Alex, A. Tiwari, Functionalized Gold Nanoparticles: Synthesis, Properties and Applications-A Review, J Nanosci Nanotechnol, 15 (2015) 1869-1894.

[7] Y.C. Yeh, B. Creran, V.M. Rotello, Gold nanoparticles: preparation, properties, and applications in bionanotechnology, Nanoscale, 4 (2012) 1871-1880.

[8] A.H. Lu, E.L. Salabas, F. Schuth, Magnetic nanoparticles: synthesis, protection, functionalization, and application, Angew Chem Int Ed Engl, 46 (2007) 1222-1244.

[9] M. Liong, J. Lu, M. Kovochich, T. Xia, S.G. Ruehm, A.E. Nel, F. Tamanoi, J.I. Zink, Multifunctional inorganic nanoparticles for imaging, targeting, and drug delivery, ACS Nano, 2 (2008) 889-896.

[10] C.N. Rao, H.S. Ramakrishna Matte, R. Voggu, A. Govindaraj, Recent progress in the synthesis of inorganic nanoparticles, Dalton Trans, 41 (2012) 5089-5120.

[11] M. Auffan, J. Rose, J.Y. Bottero, G.V. Lowry, J.P. Jolivet, M.R. Wiesner, Towards a definition of inorganic nanoparticles from an environmental, health and safety perspective, Nat Nanotechnol, 4 (2009) 634-641.

[12] S.J. Soenen, P. Rivera-Gil, J.-M. Montenegro, W.J. Parak, S.C. De Smedt, K. Braeckmans, Cellular toxicity of inorganic nanoparticles: Common aspects and guidelines for improved nanotoxicity evaluation, Nano Today, 6 (2011) 446-465.

[13] M. Bhamidipati, L. Fabris, Multiparametric Assessment of Gold Nanoparticle Cytotoxicity in Cancerous and Healthy Cells: The Role of Size, Shape, and Surface Chemistry, Bioconjug Chem, 28 (2017) 449-460.

[14] N. Hao, L. Li, F. Tang, Roles of particle size, shape and surface chemistry of mesoporous silica nanomaterials on biological systems, Int Mater Rev, 62 (2017) 57-77.

[15] M.P. Monopoli, D. Walczyk, A. Campbell, G. Elia, I. Lynch, F.B. Bombelli, K.A. Dawson, Physical-chemical aspects of protein corona: relevance to in vitro and in vivo biological impacts of nanoparticles, J Am Chem Soc, 133 (2011) 2525-2534.

[16] S. Tatur, M. Maccarini, R. Barker, A. Nelson, G. Fragneto, Effect of functionalized gold nanoparticles on floating lipid bilayers, Langmuir, 29 (2013) 6606-6614.

[17] D. Di Silvio, M. Maccarini, R. Parker, A. Mackie, G. Fragneto, F. Baldelli Bombelli, The effect of the protein corona on the interaction between nanoparticles and lipid bilayers, J Colloid Interface Sci, 504 (2017) 741-750.

[18] A. Luchini, Y. Gerelli, G. Fragneto, T. Nylander, G.K. Palsson, M.S. Appavou, L. Paduano, Neutron Reflectometry reveals the interaction between functionalized SPIONs and the surface of lipid bilayers, Colloids Surf B Biointerfaces, 151 (2017) 76-87.

[19] Y.P. Chen, Y.L. Xianyu, X.Y. Jiang, Surface Modification of Gold Nanoparticles with Small Molecules for Biochemical Analysis, Accounts Chem Res, 50 (2017) 310-319.

[20] G. Bodelón, C. Costas, J. Pérez-Juste, I. Pastoriza-Santos, L.M. Liz-Marzán, Gold nanoparticles for regulation of cell function and behavior, Nano Today, 13 (2017) 40-60.

[21] M. Grzelczak, J. Perez-Juste, P. Mulvaney, L.M. Liz-Marzan, Shape control in gold nanoparticle synthesis, Chem Soc Rev, 37 (2008) 1783-1791. 
[22] R. Sardar, A.M. Funston, P. Mulvaney, R.W. Murray, Gold nanoparticles: past, present, and future, Langmuir, 25 (2009) 13840-13851.

[23] K.N. Thakkar, S.S. Mhatre, R.Y. Parikh, Biological synthesis of metallic nanoparticles, Nanomedicine: Nanotechnology, Biology and Medicine, 6 (2010) 257-262.

[24] N. Li, P.X. Zhao, D. Astruc, Anisotropic Gold Nanoparticles: Synthesis, Properties, Applications, and Toxicity, Angew Chem Int Edit, 53 (2014) 1756-1789.

[25] H.J. Chen, L. Shao, Q. Li, J.F. Wang, Gold nanorods and their plasmonic properties, Chem Soc Rev, 42 (2013) 2679-2724.

[26] M. Cui, Y. Zhao, Q. Song, Synthesis, optical properties and applications of ultra-small luminescent gold nanoclusters, TrAC Trends in Analytical Chemistry, 57 (2014) 73-82.

[27] C. Daniela, C. Andreea, P. Rebecca, G. Alexandru Mihai, Biomedical Applications of Gold Nanoparticles, Current Topics in Medicinal Chemistry, 15 (2015) 1605-1613.

[28] H. Daraee, A. Eatemadi, E. Abbasi, S. Fekri Aval, M. Kouhi, A. Akbarzadeh, Application of gold nanoparticles in biomedical and drug delivery, Artificial Cells, Nanomedicine, and Biotechnology, 44 (2016) 410-422.

[29] M.C. Daniel, D. Astruc, Gold nanoparticles: assembly, supramolecular chemistry, quantum-sizerelated properties, and applications toward biology, catalysis, and nanotechnology, Chem Rev, 104 (2004) 293-346.

[30] N.M. Dimitriou, G. Tsekenis, E.C. Balanikas, A. Pavlopoulou, M. Mitsiogianni, T. Mantso, G. Pashos, A.G. Boudouvis, I.N. Lykakis, G. Tsigaridas, M.I. Panayiotidis, V. Yannopapas, A.G. Georgakilas, Gold nanoparticles, radiations and the immune system: Current insights into the physical mechanisms and the biological interactions of this new alliance towards cancer therapy, Pharmacol Ther, 178 (2017) 1-17.

[31] J.R. Matthews, C.M. Payne, J.H. Hafner, Analysis of Phospholipid Bilayers on Gold Nanorods by Plasmon Resonance Sensing and Surface-Enhanced Raman Scattering, Langmuir, 31 (2015) 98939900.

[32] S.E. Lee, D.Y. Sasaki, T.D. Perroud, D. Yoo, K.D. Patel, L.P. Lee, Biologically functional cationic phospholipid-gold nanoplasmonic carriers of RNA, J Am Chem Soc, 131 (2009) 1406614074.

[33] Q. Ong, Z. Luo, F. Stellacci, Characterization of Ligand Shell for Mixed-Ligand Coated Gold Nanoparticles, Acc Chem Res, 50 (2017) 1911-1919.

[34] A. Luchini, C. Irace, R. Santamaria, D. Montesarchio, R.K. Heenan, N. Szekely, A. Flori, L. Menichetti, L. Paduano, Phosphocholine-decorated superparamagnetic iron oxide nanoparticles: defining the structure and probing in vivo applications, Nanoscale, 8 (2016) 10078-10086.

[35] H. Wei, N. Insin, J. Lee, H.S. Han, J.M. Cordero, W. Liu, M.G. Bawendi, Compact zwitterioncoated iron oxide nanoparticles for biological applications, Nano Lett, 12 (2012) 22-25.

[36] K. Pombo Garcia, K. Zarschler, L. Barbaro, J.A. Barreto, W. O'Malley, L. Spiccia, H. Stephan, B. Graham, Zwitterionic-coated "stealth" nanoparticles for biomedical applications: recent advances in countering biomolecular corona formation and uptake by the mononuclear phagocyte system, Small, 10 (2014) 2516-2529.

[37] X. Ning, C. Peng, E.S. Li, J. Xu, R.D. VinluanIII, M. Yu, J. Zheng, Physiological stability and renal clearance of ultrasmall zwitterionic gold nanoparticles: Ligand length matters, APL Materials, 5 (2017) 053406.

[38] J. Choi, S. Park, Z. Stojanovic, H.S. Han, J. Lee, H.K. Seok, D. Uskokovic, K.H. Lee, Facile solvothermal preparation of monodisperse gold nanoparticles and their engineered assembly of ferritin-gold nanoclusters, Langmuir, 29 (2013) 15698-15703.

[39] M. Brust, J. Fink, D. Bethell, D.J. Schiffrin, C. Kiely, Synthesis and Reactions of Functionalized Gold Nanoparticles, J Chem Soc Chem Comm, (1995) 1655-1656.

[40] A. Luchini, G. Vitiello, F. Rossi, O. Ruiz De Ballesteros, A. Radulescu, G. D'Errico, D. Montesarchio, C. de Julian Fernandez, L. Paduano, Developing functionalized Fe3O4-Au nanoparticles: a physico-chemical insight, Phys Chem Chem Phys, 17 (2015) 6087-6097. 
[41] J.S.H.a.H.C. Benoit, Polymers and Neutron Scattering, Clarendon Press1997.

[42] Y.S. Kang, L. Kevan, Interaction of Poly(Ethylene Oxide) with the Sodium Dodecyl-Sulfate Micelle Interface Studied with Nitroxide Spin Probes, J Phys Chem-Us, 98 (1994) 7624-7627.

[43] N. Ruocco, H. Frielinghaus, G. Vitiello, G. D'Errico, L.G. Leal, D. Richter, O. Ortona, L. Paduano, How hydrophobically modified chitosans are stabilized by biocompatible lipid aggregates, J Colloid Interf Sci, 452 (2015) 160-168.

[44] L. Stella, M. Venanzi, M. Carafa, E. Maccaroni, M.E. Straccamore, G. Zanotti, A. Palleschi, B. Pispisa, Structural features of model glycopeptides in solution and in membrane phase: A spectroscopic and molecular mechanics investigation, Biopolymers, 64 (2002) 44-56.

[45] B. Venegas, M.R. Wolfson, P.H. Cooke, P.L.G. Chong, High Vapor Pressure Perfluorocarbons Cause Vesicle Fusion and Changes in Membrane Packing, Biophysical Journal, 95 (2008) 4737-4747. [46] M. Bonini, E. Fratini, P. Baglioni, SAXS study of chain-like structures formed by magnetic nanoparticles, Materials Science and Engineering: C, 27 (2007) 1377-1381.

[47] A. Luchini, R.K. Heenan, L. Paduano, G. Vitiello, Functionalized SPIONs: the surfactant nature modulates the self-assembly and cluster formation, Phys Chem Chem Phys, 18 (2016) 18441-18449. [48] G.C. Bushell, Y.D. Yan, D. Woodfield, J. Raper, R. Amal, On techniques for the measurement of the mass fractal dimension of aggregates, Advances in Colloid and Interface Science, 95 (2002) 150 .

[49] B. Gilbert, R.K. Ono, K.A. Ching, C.S. Kim, The effects of nanoparticle aggregation processes on aggregate structure and metal uptake, J Colloid Interf Sci, 339 (2009) 285-295.

[50] G. Von White, F.S. Mohammed, C.L. Kitchens, Small-Angle Neutron Scattering Investigation of Gold Nanoparticle Clustering and Ligand Structure Under Antisolvent Conditions, The Journal of Physical Chemistry C, 115 (2011) 18397-18405.

[51] J. Teixeira, Small-angle scattering by fractal systems, Journal of Applied Crystallography, 21 (1988) 781-785.

[52] S.R. Kline, Reduction and analysis of SANS and USANS data using IGOR Pro, Journal of Applied Crystallography, 39 (2006) 895-900.

[53] W.G. Wu, C.H. Huang, Kinetic studies of the micellar to lamellar phase transition of 1stearoyllysophosphatidylcholine dispersions, Biochemistry, 22 (1983) 5068-5073.

[54] K.W. Liu, S.L. Biswal, Probing insertion and solubilization effects of lysolipids on supported lipid bilayers using microcantilevers, Anal Chem, 83 (2011) 4794-4801.

[55] J. Mattai, G.G. Shipley, The Kinetics of Formation and Structure of the Low-Temperature Phase of 1-Stearoyl-Lysophosphatidylcholine, Biochimica Et Biophysica Acta, 859 (1986) 257-265.

[56] W.G. Wu, C.H. Huang, T.G. Conley, R.B. Martin, I.W. Levin, Lamellar Micellar Transition of 1-Stearoyllysophosphatidylcholine Assemblies in Excess Water, Biochemistry, 21 (1982) 59575961.

[57] M. Bergstrom, J.S. Pedersen, P. Schurtenberger, S.U. Egelhaaf, Small-angle neutron scattering (SANS) study of vesicles and lamellar sheets formed from mixtures of an anionic and a cationic surfactant, J Phys Chem B, 103 (1999) 9888-9897.

[58] A. Merlino, G. Vitiello, M. Grimaldi, F. Sica, E. Busi, R. Basosi, A.M. D'Ursi, G. Fragneto, L. Paduano, G. D'Errico, Destabilization of Lipid Membranes by a Peptide Derived from Glycoprotein gp36 of Feline Immunodeficiency Virus: A Combined Molecular Dynamics/Experimental Study, J Phys Chem B, 116 (2012) 401-412.

[59] G. Vitiello, A. Falanga, A.A. Petruk, A. Merlino, G. Fragneto, L. Paduano, S. Galdiero, G. D'Errico, Fusion of raft-like lipid bilayers operated by a membranotropic domain of the HSV-type I glycoprotein gH occurs through a cholesterol-dependent mechanism, Soft Matter, 11 (2015) 30033016.

[60] L.A. Bagatolli, LAURDAN Fluorescence Properties in Membranes: A Journey from the Fluorometer to the Microscope, in: Y. Mély, G. Duportail (Eds.) Fluorescent Methods to Study Biological Membranes, Springer Berlin Heidelberg, Berlin, Heidelberg, 2013, pp. 3-35. 
[61] T. Parasassi, G. De Stasio, A. d'Ubaldo, E. Gratton, Phase fluctuation in phospholipid membranes revealed by Laurdan fluorescence, Biophys J, 57 (1990) 1179-1186.

[62] A. Verma, F. Stellacci, Effect of surface properties on nanoparticle-cell interactions, Small, 6 (2010) 12-21.

[63] C. Montis, D. Maiolo, I. Alessandri, P. Bergese, D. Berti, Interaction of nanoparticles with lipid membranes: a multiscale perspective, Nanoscale, 6 (2014) 6452-6457. 